\title{
Chiral hexagonal cellular sandwich structures: dynamic response
}

\author{
A. Spadoni ${ }^{a}$, M. Ruzzene ${ }^{a}$, F. Scarpa ${ }^{b}$ \\ ${ }^{a}$ School of Aerospace Engineering, Georgia Institute of Technology \\ Atlanta, GA 30332 USA \\ ${ }^{b}$ Department of Mechanical Engineering, University of Sheffield \\ Sheffield, UK
}

\begin{abstract}
Periodic cellular configurations with negative Poisson's ratio have attracted the attention of several researchers because of their superior dynamic characteristics. Among the geometries featuring a negative Poisson's ratio, the chiral topology possesses a geometric complexity that guarantees unique deformed configurations when excited at one of its natural frequencies. Specifically, localized deformations have been observed even at relatively low excitation frequencies. This is of particular importance as resonance can be exploited to minimize the power required for the appearance of localized deformations, thus giving practicality to the concept. The particular nature of these deformed configurations and the authority provided by the chiral geometry, suggest the application of the proposed structural configuration for the design of innovative lifting bodies, such as helicopter rotor blades or airplane wings. The dynamic characteristics of chiral structures are here investigated through a numerical model and experimental investigations. The numerical formulation uses dynamic shape functions to accurately describe the behavior of the considered structural assembly over a wide frequency range. The model is used to predict frequency response functions, and to investigate the occurrence of localized deformations. Experimental tests are also performed to demonstrate the accuracy of the model and to illustrate the peculiarities of the behavior of the considered chiral structures.
\end{abstract}

Keywords: Chiral structures, localized deformations, Negative Poisson's ratio

\section{INTRODUCTION}

In recent years, cellular structures have seen increased interest and applications due to their excellent mechanical properties and low weight characteristics. The application of stronger and lighter structural assemblies and materials is particularly relevant to the aircraft and aerospace industries, although many other applications can benefit from advances in materials and components. Ongoing interests in cellular structures and improvements in the manufacturing processes have contributed to the study and development of cellular solids of innovative shapes and topologies. Much interest has recently centered on cellular assemblies featuring a negative Poisson's ratio behavior, also known as "auxetic" . ${ }^{1}$ Materials having auxetic characteristics include special subsets of foams,$^{2}$ long fiber composites ${ }^{3}$ microporous polymers,${ }^{1}$ as well as honeycombs ${ }^{4}$ The mechanical properties of these alternatives to solid components have thoroughly been investigated by ${ }^{5-8}$ among others. Among the proposed innovative geometries, chiral topologies ${ }^{9,10}$ feature a unique geometric configuration which can be exploited for the design of innovative structural components. Previous investigations by the authors have in fact shown how this topology can be exploited for the design of sandwich panels with interesting structural acoustic behavior, ${ }^{11}$ and as an alternative honeycomb configuration with superior flat-wise compression strength.$^{12}$

In the current work the dynamic properties of truss-core sandwich beams with chiral core are investigated numerically and experimentally. The considered configuration is designed to generate deformed shapes, where the deformations are mostly localized in limited regions of the structure. These localized deformed shapes correspond to mode shapes and in principle they can be obtained through the excitation of the component at one of its resonant frequencies. The feasibility of these concepts is investigated numerically on models such as the

Send correspondence to:

M. Ruzzene: E-mail: massimo.ruzzene@aerospace.gatech.edu, Telephone: 14048943078 


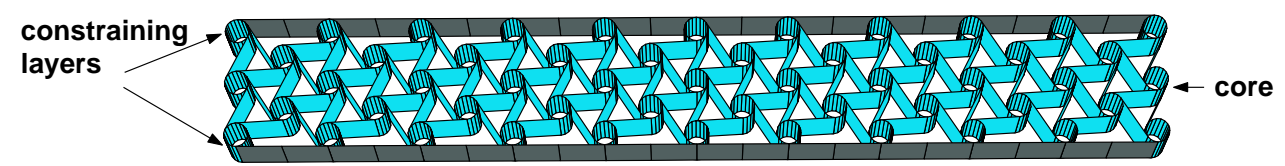

Figure 1. Truss-core beam with chiral honeycomb core.

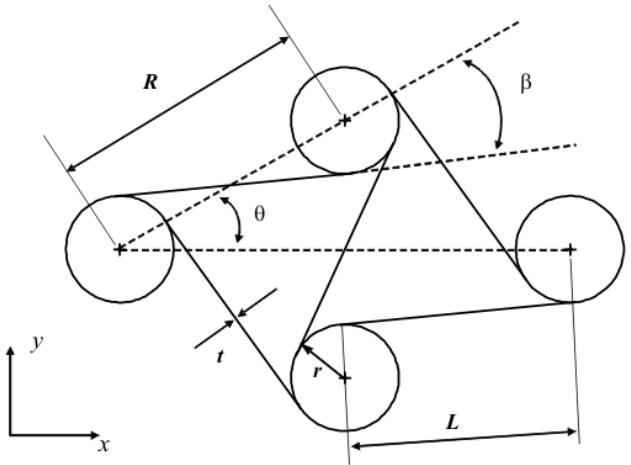

Figure 2. Chiral geometry of a core unit cell.

one shown in Figure 1. Numerical simulations are formulated by employing dynamic-shape functions, derived directly from the distributed parameter model of beam elements. Finally, the analytical results are compared to experimental measurements in an effort to validate the behavior observed in the numerical simulations.

The paper is organized as follows: section 1 provides a brief introduction, and a background for the considered study, and section 2 gives an overview of the considered assembly and describes the geometry of the chiral topology. Section 3 presents the distributed parameter model and its implementation, while section 4 offers an overview of the experimental setup and equipment used. The experimental and analytical results are presented and compared in section 5. Finally, section 6 summarizes the findings of the study and provides recommendations for future work.

\section{CONCEPT AND CONSIDERED CONFIGURATIONS}

The dynamic performance of assemblies of the kind shown in Figure 1 is here investigated. The considered structure features a truss-type core with elements arranged according to a chiral topology, constrained by two uniform layers. The unit cell defining the chiral core is depicted in Figure 2. The unit cell is defined by ribs or walls connecting nodes or cylinders. The distance between the nodes is denoted as $R$, while the length of the ribs tangent to the nodes is $L$. Also, $r$ and $t$ are respectively the radius of the nodes and the wall thickness of all components. Finally, the angle between the lines connecting the node centers is denoted as $\theta$, while the angle between the line connecting the nodes centers and the ribs is $\beta$. The following relationships defining the cell geometry hold ${ }^{10}$ :

$$
\sin \beta=\frac{2 r}{R}, \quad \tan \beta=\frac{2 r}{L}
$$

For a hexagonal geometric layout $\theta$ is equal to $30^{\circ}$. Varying $L, R$ and $t$, produces a variety of core configurations and significantly alters the mechanical properties of the obtained assembly. The parameters $L, R$ and $t$, are here considered as design variables which can be properly selected in order to obtain the localized dynamic deformations here investigated. 


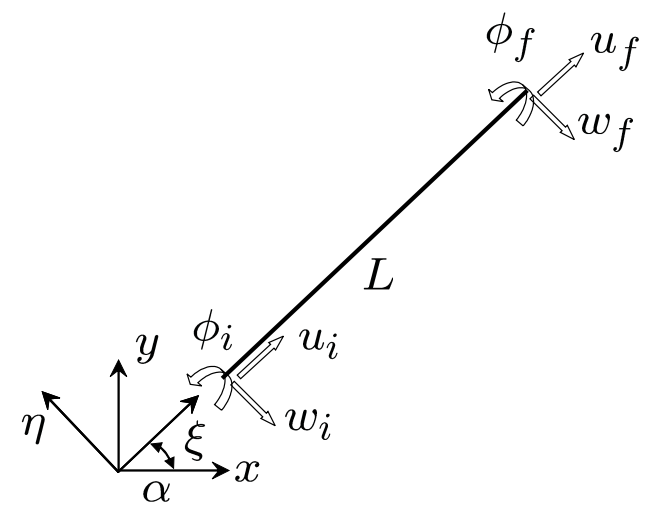

Figure 3. Global and local reference systems and element degrees of freedom.

\section{SPECTRAL FINITE ELEMENT MODEL}

The dynamic behavior of the considered structural assembly is predicted by a Spectral Finite Element (SFE) model. The SFE method describes the behavior of each element composing the considered mesh through dynamic equations derived from distributed parameter models ${ }^{13,14}$ This allows the accurate prediction of the dynamic response of the structure over a wide frequency range, without having to refine the discretization as frequency increases. The considered sandwich beam is composed of rigidly connected straight beam elements, and thus is modeled as a frame structure, with elements oriented in the plane $x, y$ according to the core topology. The dynamic behavior of each element is described in the local reference system $\mathcal{L}=(\xi, \eta)$, rotated with respect to the global reference system $\mathcal{G}=(x, y)$ of a generic angle $\alpha$ (see Figure 3 ). The circular nodes are approximated as a sequence of straight beams. The resulting model contains elements of various lengths, some of which are very small. A Timoshenko beam model is thus considered to prevent lack of accuracy corresponding to the description of relatively short members through Euler-Bernoulli theory.

\subsection{Distributed parameter model in the local reference system}

The equations of motion and boundary conditions governing the longitudinal and transverse vibrations of a beam element can be derived by applying Hamilton's principle ${ }^{15}$ :

$$
\int_{t_{1}}^{t_{2}} \delta(T-U+W) d t=0
$$

where $\delta($.$) denotes the first variation, t_{1}$ and $t_{2}$ are the initial and final time, $T$ and $U$ are respectively the kinetic and the strain energy of the beam, and $W$ is the work done by the external forces. Each element is considered as a Timoshenko beam, which includes axial degrees of freedom also. Accordingly, in the local reference system, the beam's strain energy can be expressed as:

$$
U=\frac{1}{2} \int_{0}^{L}\left[E A u_{, \xi}^{2}+E I \phi_{, \xi}^{2}+\kappa A G\left(w_{, \xi}-\phi\right)^{2}\right] \mathrm{d} \xi,
$$

where $E, G$ are the Young's and shear moduli of the beam material, $A, I$ are the area and the second moment of area of the beam cross section, and $\kappa$ is the shear coefficient. Also, in equation $(3), u=u(\xi, t), w=w(\xi, t)$ denote the axial and transverse deflection of the beam, while $\phi=\phi(\xi, t)$ is the rotation of the cross section at location $\xi$ and time $t$. Finally, the following notation $(\diamond)_{, \xi}=\frac{\partial(\diamond)}{\partial \xi}$ is used to denote partial differentiation.

The kinetic energy is given by

$$
T=\frac{1}{2} \int_{0}^{L}\left[\rho A\left(u_{, t t}^{2}+w_{, t t}^{2}\right)+\rho I \phi_{, t t}^{2}\right] \mathrm{d} x
$$


where $\rho$ is the density of the beam material.

Substituting $U$ and $T$ in equation (2), assuming the absence of external forces ( $W=0$ ), and performing the variations gives a set of three differential equations describing the beam's longitudinal and transverse vibration. For harmonic motion at frequency $\omega$ the equations of motion are:

$$
\begin{aligned}
E A u_{, \xi \xi}+\rho A \omega^{2} u & =0 \\
\kappa G A\left(w_{, \xi \xi}-\phi_{, \xi}\right)+\rho A \omega^{2} w & =0 \\
E I \phi_{, \xi \xi}+\kappa G A\left(w_{, \xi}-\phi\right)+\rho I \omega^{2} \phi & =0
\end{aligned}
$$

\subsection{Dynamic Stiffness Matrix in Local Coordinates}

Equations (5) can be rewritten in matrix form as follows:

$$
\boldsymbol{z}_{, \xi}(\xi)=\boldsymbol{A} \boldsymbol{z}(\xi),
$$

where $\boldsymbol{A}$ is a matrix of constant coefficients, and where

$$
\boldsymbol{z}=\left[\begin{array}{llllll}
u & u_{, \xi} & w & \phi & w_{, \xi} & \phi_{, \xi}
\end{array}\right]^{T}
$$

is the state vector describing the axial and bending behavior of the considered beam element at frequency $\omega$. In the current development, lower case bold letters indicate vectors, while capital bold case letters indicate matrices.

A general solution of equation (6) can be expressed as:

$$
\boldsymbol{z}(\xi)=e^{\boldsymbol{A} \xi} \boldsymbol{z}(0),
$$

which relates the vector $\boldsymbol{z}$ at the generic location $\xi$ to that of $\xi=0$. An alternative expression for the state vector can be introduced to contain generalized displacements and stress resultants at the considered location. A vector $\boldsymbol{y}$ can be defined as:

$$
\boldsymbol{y}=\left[\begin{array}{llllll}
u & w & \phi & N & V & M
\end{array}\right]^{T},
$$

where:

$$
N(\xi)=E A u_{, \xi}, V(\xi)=\kappa G A\left(w_{, \xi}-\phi\right), M(\xi)=E I \phi_{, \xi}
$$

are respectively the axial resultant, the shear force and the bending moment at location $\xi$. The vector $\boldsymbol{y}$ and $\boldsymbol{z}$ are simply related through the following expression:

$$
\boldsymbol{y}(\xi)=\boldsymbol{G} \boldsymbol{z}(\xi),
$$

where $\boldsymbol{G}$ is a matrix containing the material and cross sectional properties of the beam element. Equations (8) and (11) can be used to obtain a relation between state vectors at two locations on the element:

$$
\begin{aligned}
& \boldsymbol{y}(\xi)=\boldsymbol{G} e^{\boldsymbol{A} \xi} \boldsymbol{G}^{-1} \boldsymbol{y}(0), \\
& \boldsymbol{y}(\xi)=\boldsymbol{T}(\xi) \boldsymbol{y}(0),
\end{aligned}
$$


where $\boldsymbol{T}(\xi)$ is the "transfer matrix" of the beam element calculated at location $\xi$. The transfer matrix can be used to relate generalized displacements and forces at the two ends of the beam element:

$$
\boldsymbol{y}(L)=\boldsymbol{T}(L) \boldsymbol{y}(0)
$$

Equation (13) then can be expanded as follows:

$$
\left(\begin{array}{c}
\boldsymbol{u}_{i} \\
\boldsymbol{f}_{i}
\end{array}\right)=\left(\begin{array}{ll}
\boldsymbol{T}_{11} & \boldsymbol{T}_{12} \\
\boldsymbol{T}_{21} & \boldsymbol{T}_{22}
\end{array}\right)\left(\begin{array}{c}
\boldsymbol{u}_{f} \\
\boldsymbol{f}_{f}
\end{array}\right),
$$

where $\boldsymbol{u}_{i}, \boldsymbol{u}_{f}$ and $\boldsymbol{f}_{i}, \boldsymbol{f}_{f}$ respectively are the generalized displacements and forces at the initial and final node. Equation (14) can be rearranged to obtain:

$$
\boldsymbol{f}_{e}^{\mathcal{L}}=\boldsymbol{K}_{d_{e}}^{\mathcal{L}} \boldsymbol{u}_{e}^{\mathcal{L}}
$$

where $\boldsymbol{f}_{e}^{\mathcal{L}}=\left(\begin{array}{ll}\boldsymbol{f}_{i} & \boldsymbol{f}_{f}\end{array}\right)^{T}, \boldsymbol{u}_{e}^{\mathcal{L}}=\left(\begin{array}{ll}\boldsymbol{u}_{i} & \boldsymbol{u}_{f}\end{array}\right)^{T}$, and where $\boldsymbol{K}_{d_{e}}^{\mathcal{L}}$ is the dynamic stiffness matrix of the element, which is obtained as:

$$
\boldsymbol{K}_{d_{e}}^{\mathcal{L}}=\left(\begin{array}{cc}
-\boldsymbol{T}_{12}^{-1} \boldsymbol{T}_{11} & \boldsymbol{T}_{12}^{-1} \\
\boldsymbol{T}_{12}+\boldsymbol{T}_{22} \boldsymbol{T}_{12}^{-1} \boldsymbol{T}_{11} & \boldsymbol{T}_{22} \boldsymbol{T}_{12}^{-1}
\end{array}\right)
$$

In equation (15), the subscript $e$ denotes vectors pertaining to the element, while superscript $\mathcal{L}$ indicates that the expression is obtained in the local reference system $\mathcal{L}=(\xi, \eta)$.

This frequency-domain, finite-element formulation, denoted as "spectral", ${ }^{13}$ allows for an accurate prediction of the dynamic response of the global structure by using a reduced number of elements, since, as opposed to traditional finite elements, the number of elements does not need to be increased to fully capture the dynamic response at high frequencies. One should however note that the evaluation of the shape functions from the continuous beam model requires the evaluation of an exponential matrix, which at extremely high frequencies may become affected by numerical errors that limit the accuracy of the analysis. These numerical difficulties can be circumvented by reducing the elements' length, thus considering finer meshes. Regardless of such limitations, the high frequency behavior can be captured with a substantially reduced number of elements with respect to conventional formulations.

\subsection{Dynamic shape functions}

The interpolation functions which express the generalized displacements of points within the element as a function of the nodal degrees of freedom can be expressed using the transfer function formulation (equation (12)). According to equation (12), the generalized displacements at location $\xi$ can be expressed as:

$$
\boldsymbol{u}(\xi)=\boldsymbol{T}_{11}(\xi) \boldsymbol{u}_{i}+\boldsymbol{T}_{12}(\xi) \boldsymbol{f}_{i}
$$

and in particular the displacements for $\xi=L$ are given as:

$$
\boldsymbol{u}_{f}=\boldsymbol{T}_{11}(L) \boldsymbol{u}_{i}+\boldsymbol{T}_{12}(L) \boldsymbol{f}_{i}
$$

From equation (18), one obtains:

$$
\boldsymbol{f}_{i}=\boldsymbol{T}_{12}^{-1}(L)\left(\boldsymbol{T}_{11}(L) \boldsymbol{u}_{i}-\boldsymbol{u}_{f}\right)
$$


which can be substituted into equation (17) to give:

$$
\begin{aligned}
& \boldsymbol{u}(\xi)=\left(\boldsymbol{T}_{11}(\xi)+\boldsymbol{T}_{12}(\xi) \boldsymbol{T}_{12}^{-1}(L) \boldsymbol{T}_{11}(L)-T_{12}(\xi) \boldsymbol{T}_{12}^{-1}(L)\right) \boldsymbol{u}_{e}^{\mathcal{L}}, \\
& \boldsymbol{u}(\xi)=\boldsymbol{N}(\xi) \boldsymbol{u}_{e}^{\mathcal{L}},
\end{aligned}
$$

where $\mathbf{N}(\xi)$ is the matrix of the dynamic interpolation functions. The dynamic stiffness matrix for the element is obtained from the beam's distributed parameter model for harmonic motion at frequency $\omega$. Within the validity of Timoshenko approximations, the model reproduces the exact displacements of the considered element. Accordingly, a single finite element is sufficient to fully characterize the response of a beam.

\subsection{Dynamic stiffness matrix in the global reference system}

The components of the beam's longitudinal and transverse displacements $u, w$ in the global reference system $\mathcal{G}=(x, y)$ are given in terms of the rotation angle $\alpha$ in Figure 3. Accordingly, the vector of nodal displacements $\boldsymbol{u}_{e}^{\mathcal{G}}$ in the global reference system is related to the local nodal displacements vector $\boldsymbol{u}_{e}^{\mathcal{L}}$ through a rotation matrix $\boldsymbol{R}=\boldsymbol{R}(\alpha)$ :

$$
\boldsymbol{u}_{e}^{\mathcal{L}}=\boldsymbol{R} \boldsymbol{u}_{e}^{\mathcal{G}}
$$

A similar relation holds for the vector of the generalized forces. The dynamic stiffness formulation in the global reference system gives:

$$
\boldsymbol{f}_{e}^{\mathcal{G}}=\boldsymbol{K}_{d_{e}}^{\mathcal{G}} \boldsymbol{u}_{e}^{\mathcal{G}}
$$

where $\boldsymbol{K}_{d_{e}}^{\mathcal{G}}=\boldsymbol{R}^{T} \boldsymbol{K}_{d_{e}}^{\mathcal{L}} \boldsymbol{R}$, with the superscript $\mathcal{G}$ indicating reference to the global reference system. Assembly of equation (22) for all the elements of the truss-core beam yields the discretized equation of motion for the considered structure, which has the well known form:

$$
\boldsymbol{f}=\boldsymbol{K}_{d} \boldsymbol{u}
$$

where $\boldsymbol{u}, \boldsymbol{f}, \boldsymbol{K}_{d}$ are the vector of the degrees of freedom of the entire structure, the vector of the equivalent external nodal loads, and the assembled dynamic stiffness matrix. Solution of the equation for an assigned set of external loads at given frequency yields the amplitude of the displacements at each node. The displacements within each element then can be obtained through the general elemental solution and the dynamic interpolation functions described by equation (20).

\section{EXPERIMENTAL MODEL}

\subsection{Overview}

A proof-of-concept truss-core beam has been built with the objective of demonstrating the dynamic behavior of chiral assemblies here investigated. Geometry and materials for the experimental specimen have been selected with the goal of maintaining simplicity of fabrication while utilizing off-the-shelf components. 

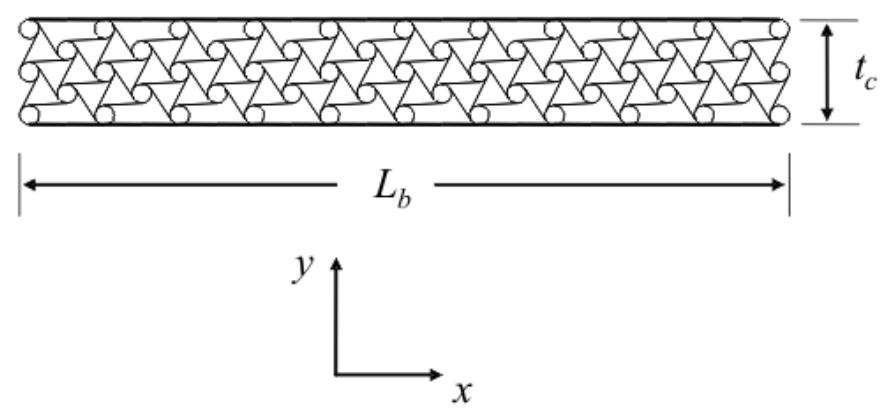

Figure 4. Truss core beam with chiral honeycomb core.

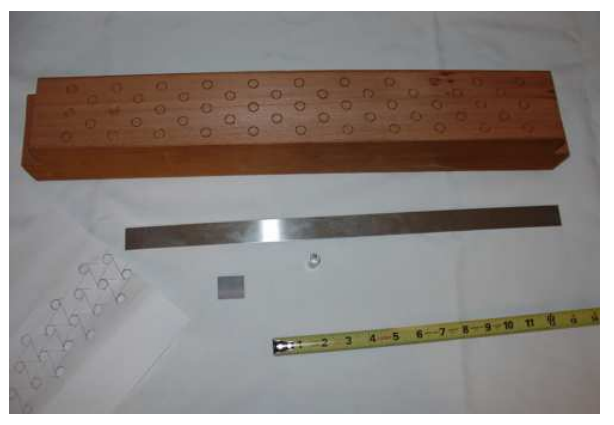

(a)

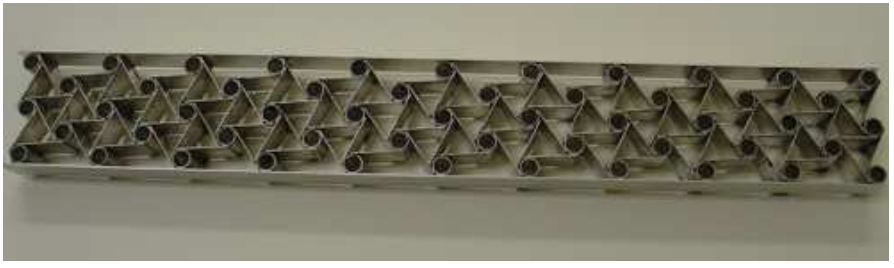

(b)

Figure 5. Wooden template (a) and completed assembly (b).

\subsection{Truss-core fabrication}

The radius $r$ of the nodes, the length of the ribs, and the wall thickness of both ribs and nodes are chosen to allow bending deformation of the ribs with low deformation of the nodes. This is a condition necessary to ensure the peculiar "unfolding" behavior of deformed chiral honeycombs. ${ }^{9,10}$ Both the core ribs and the constraining layers (see Figure 4) are fabricated from standard alloy 6061 T6 aluminum sheet (Young's modulus $E=7.0$ GPa, density $\rho=2700 \mathrm{Kg} / \mathrm{m}^{3}$, Poisson's ratio $\left.\nu=0.33\right)$. The ribs are $2.90 \mathrm{~cm}$ long, and have a wall thickness of $0.81 \mathrm{~mm}$, which corresponds to the standard thickness of 0.032 in aluminum sheets. The nodes are fabricated from $7 / 16$ in aluminum tubing with wall thickness $t=0.89 \mathrm{~mm}(0.035 \mathrm{in})$. The top and bottom constraining layers are $49.24 \mathrm{~cm}$ long and their wall thickness is equal to $0.81 \mathrm{~mm}$. Finally, the out-of-plane thickness of the structure is equal to $1 \mathrm{in}$. Given these dimensions and the geometry shown in Figure 2, the parameter $L$ becomes equal to $2.59 \mathrm{~cm}$, while the distance between the nodes $R$ is equal to $2.82 \mathrm{~cm}$. Due to the thickness of the ribs, the ratio $L / R$ decreases from a nominal value of 0.919 to 0.892 .

The complete truss core beam is composed of 53 nodes and 112 ribs. The overall dimensions of the structure are shown in Figure 4 are $L_{b}=0.5 \mathrm{~m}$, and $t_{c}=6.9 \mathrm{~cm}$. The experimental specimen was assembled by first constructing a wooden template to ensure correct positioning of the nodes (see Figure 5a). The nodes were first placed in the template at the correct locations. Then the constraining layers and ribs were welded to the nodes using the $\mathbf{3} \boldsymbol{M}$ epoxy adhesive $D P-190$ to obtain the completed assembly shown in Figure $5 \mathrm{~b}$.

\subsection{Experimental Approach}

The onset of localized deformations is a phenomenon which takes place at resonant frequencies of the complete assembly. Hence, it is important to minimize added or "external" masses, such as actuators or measuring devices in that they may change the dynamic response of the truss-core beam. This challenge is addressed by employing a scanner-laser vibrometer system to investigate the dynamic behavior of the chiral truss-core beam. 


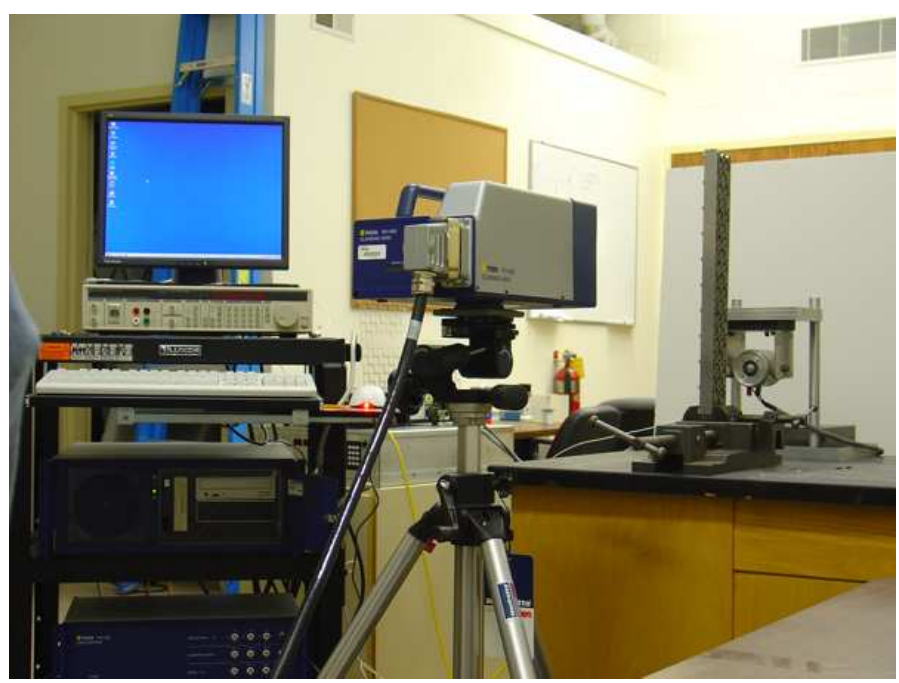

Figure 6. Experimental setup and testing equipment.

The structure is constrained at one end by two vices which rigidly clamp both the core and the constraining layers. The measurement setup is shown in Figure 6, and it consists of the Polytec PSV-400 laser scanning head, the Polytec PSV-400 M2 data acquisition system and signal processing, a LDS V203 shaker, and a 10-lb PCB Piezotronics force transducer, mounted on the shaker's stinger. In the experiments, the shaker excites the structure according to a prescribed forcing function. The vibration of the structure is characterized by measuring the velocity of points belonging to a 1281-point measurement grid on the constraining layer facing the laser head.

\section{EXPERIMENTAL RESULTS AND DISCUSSION}

The structural assembly analyzed in the current paper is excited by a random load exerted by the shaker on the layer opposite the one facing the scanning head (Figure 6). The excitation is applied over a $0-2000 \mathrm{~Hz}$ frequency range, which covers a wide portion of the modal behavior of the structure. The response of the trusscore beam is evaluated in terms of frequency-response function (FRF). The FRF for the free-tip displacement is plotted in Figure 7, where it is compared with the numerical predictions. The measured response at low frequencies compares well with the numerical simulations. Above $1600 \mathrm{~Hz}$ however, the viscous effects introduced by the epoxy adhesive become dominant and the disagreement between the analytical and experimental models becomes considerable. In the model, structural damping is introduced by defining the Young's modulus as $E=7.1 \times 10^{10}(1+i \cdot \eta)$, where $\eta$ is chosen to be 0.02 for the core components and equal to 0 for the constraining layers.

As discussed in ${ }^{11}$ and originally pointed out by ${ }^{16}$ the behavior of truss-core beams can be divided into frequency regions which depend on geometry and material properties. In the low frequency region, the behavior of truss-core beams can be treated as that of an equivalent homogeneous beam. The deformed configurations for the low frequency region are depicted in Figure 8. The deformed configurations obtained from the analytical model are depicted on the left side, while those from the experimental model are shown on the right side. The red line plotted over each deformed shape symbolizes the undeformed edge, highlighting the nodes, or zero-axis crossings of the deformed profiles. Figure 8 presents good agreement between experimental and SFE models. The uniform-beam-like behavior, however, is interrupted by intracell resonance, as the excitation frequency increases. This response boundary takes place because wavelengths become of the order of the length of the core elements ${ }^{17}$ The phenomenon of intracell resonance is demonstrated by the deformed profiles shown in Figure 8 . Particularly for values of $\Omega$ around $1550 \mathrm{~Hz}$, the core appears nearly undeformed, while the constraining layers produce localized deformations. Specifically, at $\Omega=1546 \mathrm{~Hz}$ the experimental model produces a marked kink at the free end of the bean, while the rest of the constraining layer is much less deformed. The deformed shape of 


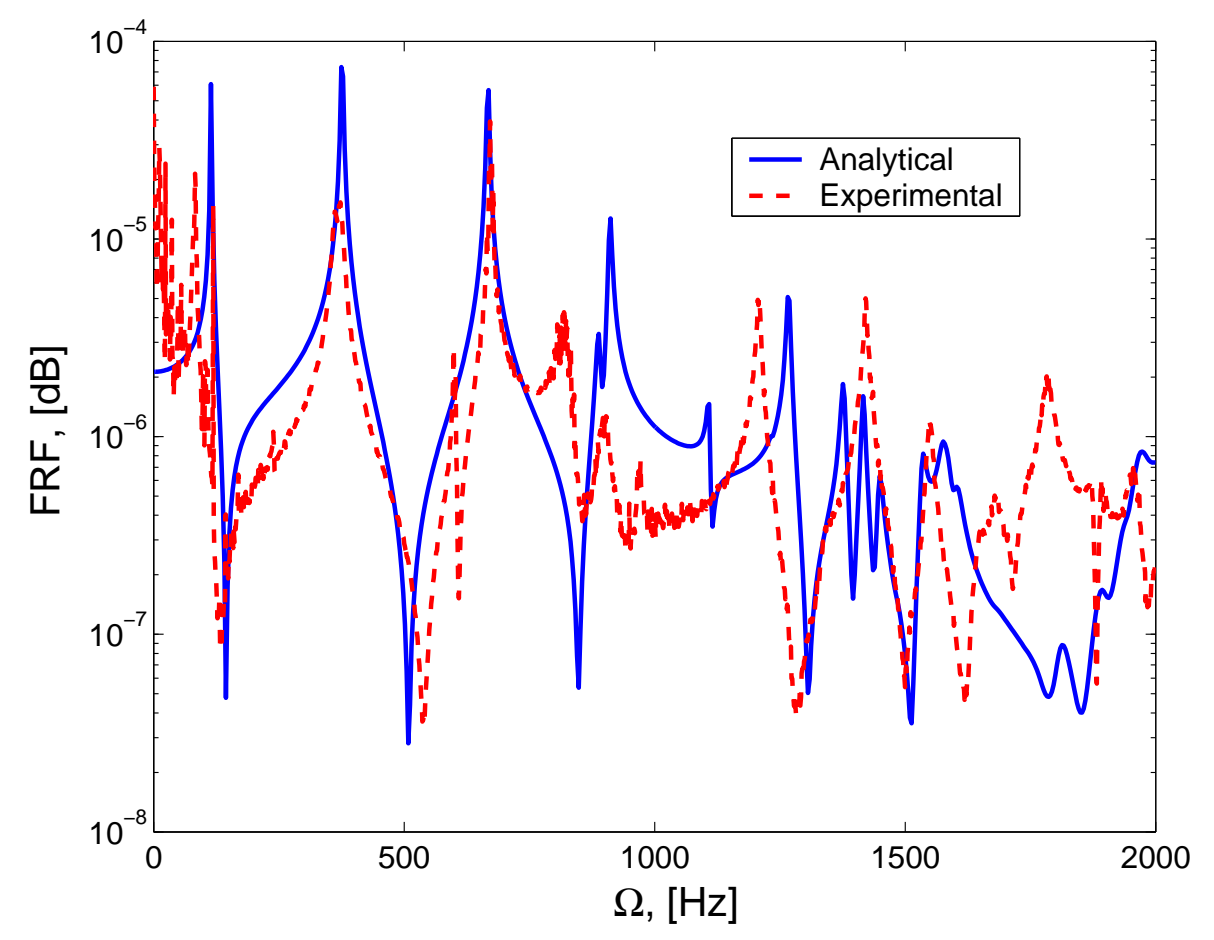

Figure 7. Frequency-response function of the analytical and experimental models.

the constraining layer obtained from the SFE model for $\Omega=1536 \mathrm{~Hz}$, on the other hand, appears much more corrugated. Overall however, agreement between the SFE and experimental models is very good at frequencies up to approximately $1110 \mathrm{~Hz}$ for the particular material and geometric properties defining the truss-core beam presented in the current paper. At high frequencies, the analytical model only qualitatively agrees with the SFE model. While the analytical FRF predicts the structure's behavior fairly well up to $1600 \mathrm{~Hz}$, the deformed shapes at such excitation frequencies for the analytical model appear to be much less damped than the ones observed experimentally.

\section{CONCLUSIONS AND FUTURE WORK}

In the current paper, the dynamic behavior of a truss-core beam composed of a chiral-honeycomb core and constraining layers is presented. A proof-of-concept model has been hand-built to validate the results obtained from numerical models. The presented results show how the numerical model qualitatively agrees with the experimental results. At low frequencies, however, the agreement has both qualitative and quantitative nature. At such excitation regimes, the truss-core beam assembly behaves similarly to a uniform beam, presenting bending modes. At higher frequencies the influence of the epoxy adhesive becomes significant, and the effects of such material discontinuity in the model are not taken into account by the numerical formulation. In this frequency region however, the deformed shapes of the analytical model qualitatively agree with those of the experimental model.

The results presented in this paper suggest the potential of the chiral honeycomb in dynamic response applications. In particular, it has been here demonstrated that, at specific excitation frequencies the resonance of the presented truss-core beam produces localized deformations far from the excitation region. The implications of such capability are the ability to actuate a structure with low energy requirements. The investigation on the proof-of-concept model, however, should be followed by a more-accurately fabricated model of the truss-core beam. 

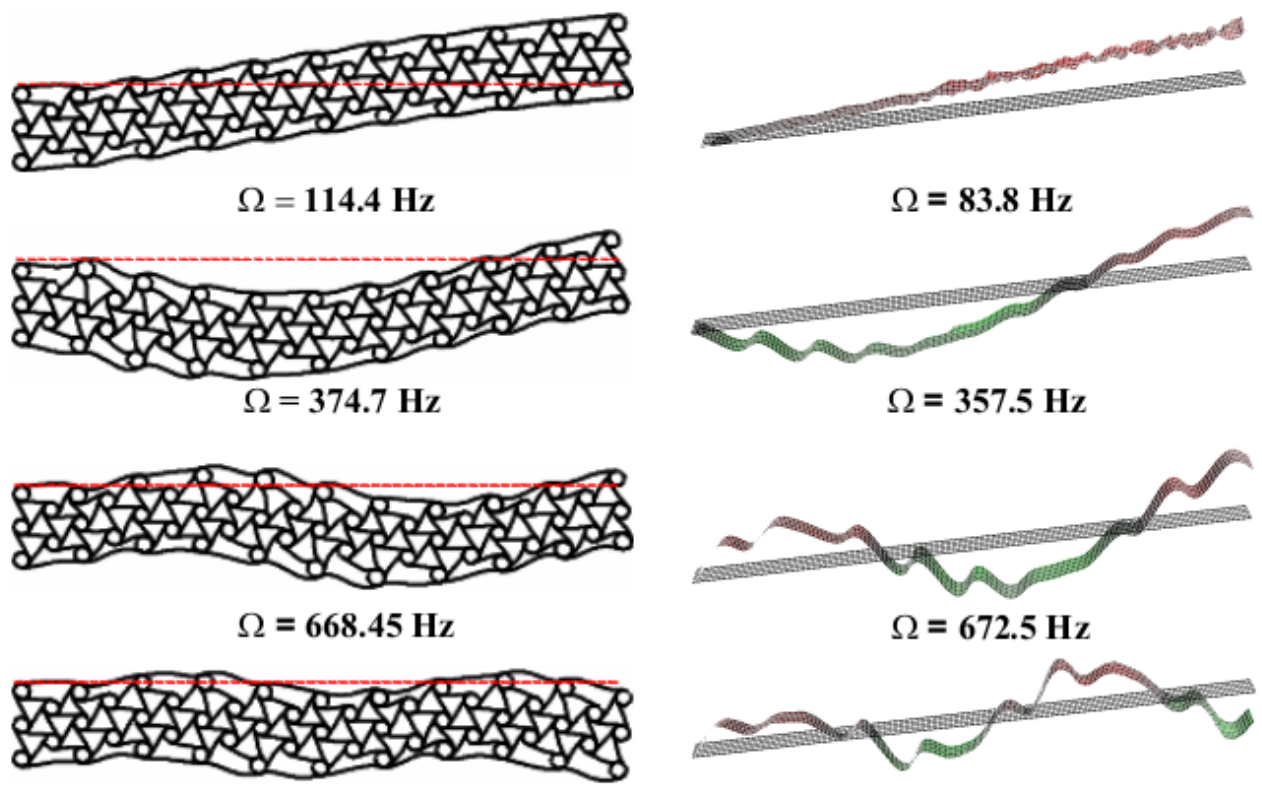

$\Omega=912.06 \mathrm{~Hz}$

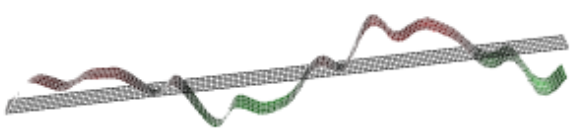

$\Omega=895.0 \mathrm{~Hz}$

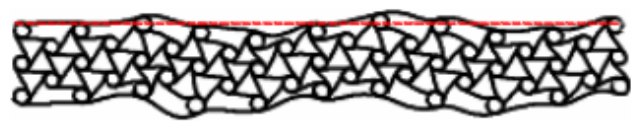

$\Omega=1108.96 \mathrm{~Hz}$

$\Omega=1200.0 \mathrm{~Hz}$

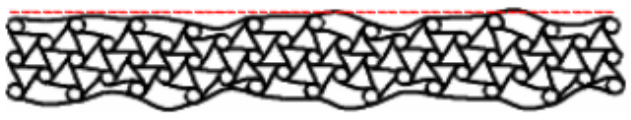

$\Omega=1375.94 \mathrm{~Hz}$

$\Omega=1410 \mathrm{~Hz}$

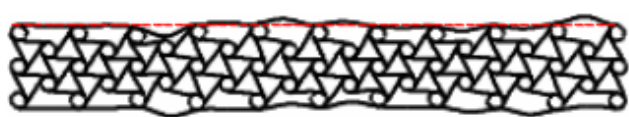

$\Omega=1536.13 \mathrm{~Hz}$

$\Omega=1546.0 \mathrm{~Hz}$

Figure 8. Experimental and analytical deformed shapes. 


\section{ACKNOWLEDGMENTS}

This work is supported by the grant W911NF0410141 from the Army Research Office. The authors wish to thank Dr. Gary Anderson, program manager and technical monitor, for his support and for his invaluable technical inputs.

\section{REFERENCES}

1. R. L. Lakes J. Mat. Sci. 26, p. 2287, 1991.

2. R. L. Lakes Science 235, p. 1038, 1987.

3. K. L. Alderson, A. Alderson, and K. E. Evans J. Strain Analysis 32(3), p. 201, 1996.

4. F. Scarpa and G. Tomlinson J. Sound Vibration 230(3), p. 45, 2000.

5. L. J. Gibson and M. F. Ashby, Cellular Solids: Structure Properties, Cambridge University Press, Cambridge, UK, 1997.

6. J. Zang and M. F. Ashby, he Out-of-Plane Properties of Honeycombs, Cambridge University Press, Cambridge, UK, 1991.

7. A. G. Evans, J. W. Hutchinson, N. A. Fleck, M. F. Ashby, and H. N. G. Wadley, "The topological design of multifunctional cellular metals," Progress in Materials Science 46, pp. 309-327, 2001.

8. N. Wicks and J. Hutchinson, "Optimal truss plates," International Journal of Solids and Structures 38, pp. 5165-5183, 2001.

9. R. S. Lakes, "Deformation mechanisms in negative poisson's ratio materials: Structural aspects," J. Mat. Sci. 26, pp. 2287-2292, 1991.

10. D. Prall and R. S. Lakes, "Properties of a chiral honeycomb with poisson's ratio of -1," Int. J. of Mechanical Sciences 39, pp. 305-314, 1996.

11. A. Spadoni and M. Ruzzene, "Structural and acoustic behavior of chiral trusscore beams," in Procedings of the ASME Noise Control and Acoustic Division (IMECE 2004), NCA (31), 2004.

12. A. Spadoni, M. Ruzzene, and F. Scarpa, "Global and local linear buckling behavior of a chiral cellular structure," Accepted Physical Status Solidi (b) , 2005.

13. J. F. Doyle, Wave Propagation in Structures, 2nd Edition, Springer Verlag, New York, NY, 1997.

14. J. F. Doyle, "A spectrally-formulated finite element for longitudinal wave propagation," Int. Journal of Analytical and Experimental Modal Analysis 3, pp. 1-5, 1988.

15. M. Petyt, Introduction to Finite Element Vibration Analysis, Cambridge University Press, New York, NY, 1990.

16. M. El-Raheb, "Frequency response of a two-dimensional truss-like period panel," Journal of the Acoustic Society of America 101(6), pp. 3457-3465, 1997.

17. A. K. Noor, "Continuum modeling for repetitive lattice structures," Applied Mechanics Reviews 41(7), pp. $285-296,1988$. 